\title{
Spectrophotometric Determination of Aminophenol Isomers Via Oxidative Coupling Reaction with 4-Aminoantipyrine
}

\author{
Reem A. Al-Luhaiby ${ }^{1 *}$, Mohammed S. Al-Enizzi ${ }^{2}$ \\ ${ }^{1,2}$ Department of Chemistry, College of Education for Girls, University of Mosul, Mosul, Iraq \\ E-mail: ${ }^{1 *}$ remawy92@gmail.com, ${ }^{2}$ mohammedsalim1977@yahoo.com
}

(Received January 17, 2020; Accepted March 16, 2020; Available online September 01, 2020)

DOI: 10.33899/edusj.2020.126543.1041, ( ) 2020, College of Education for Pure Science, University of Mosul.

This is an open access article under the CC BY 4.0 license (http://creativecommons.org/licenses/by/4.0/).

\begin{abstract}
:
A spectrophotometric method has been developed for the determination of aminophenol isomers (o-aminophenol, m-aminophenol and p-aminophenol). The method is based on oxidative coupling reaction of these compounds with 4-aminoantipyrine (4-AAP) in the presence of cupper sulphate as oxidizing agent in alkaine medium forming a reddish brown colour. The products show maximum absorption at $440 \mathrm{~nm}, 480 \mathrm{~nm}$ and $445 \mathrm{~nm}$ for o-aminophenol, m-aminophenol and p-aminophenol respectively. The molar absorptivities are $8.632 \times 10^{3}, 9.33 \times 10^{3}$ and $9.1449 \times 10^{3} 1 . \mathrm{mol}^{-1} . \mathrm{cm}^{-1}$ for concentrations obeyed Beer's law in the range 1-20, 1-24 and 1-7 $\mu \mathrm{g} \cdot \mathrm{ml}^{-1}$ for the above compounds respectively. The average recovery was ranged between $98.38 \%$ and $101.01 \%$ with relative standard deviation $<1.6$ for all the studied compounds. The 4-AAP products were formed in the ratio of 1:2 aminophenol isomers : 4-AAP. The stability constant of the products was $7.4 \times 10^{8}, 3.27 \times 10^{8}$ and $9.94 \times 10^{7} 1^{2} \cdot \mathrm{mol}^{-2}$ for o-aminophenol, m-aminophenol and p-aminophenol products respectively indicating the good stability of these products.
\end{abstract}

Key words: Oxidative coupling; Spectrophotometry; o-aminophenol; m-aminophenol; p-aminophenol, 4-aminoantipyrine

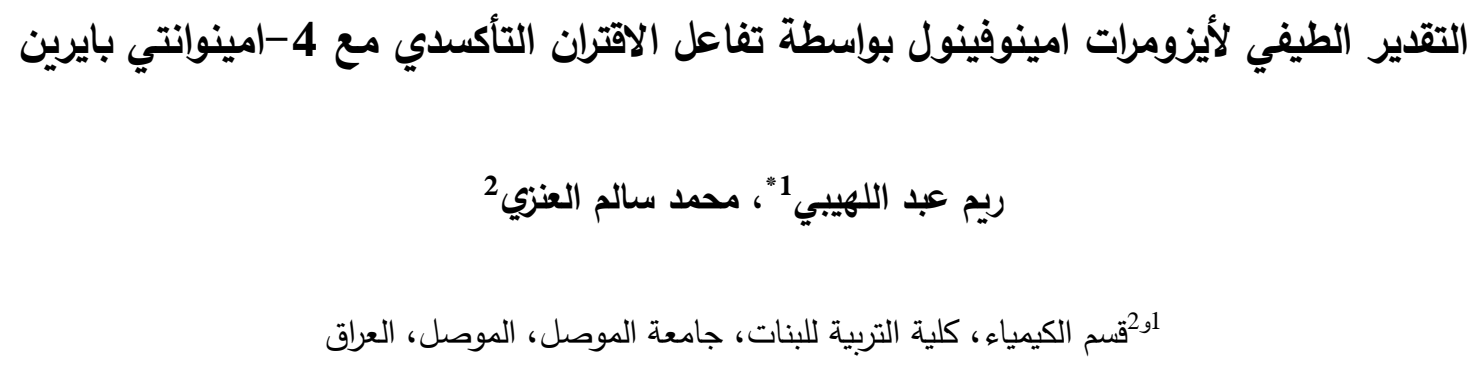




\section{Journal of Education and Science (ISSN 1812-125X), Vol: 29, No: 3, 2020 (68-84)}

الخلاصة

تم تطـوير طريقة طيفية لتقدير ايزومرات امينوفينول (أورثو-امينوفينول، ميتا-امينوفينول وبارا-امينوفينول) تعتمد على تفاعل الاقتران التأكسي لهذه المركبات مع 4-امينوانتي بايرين (4-AAP) بوجود كبريتات النحاس كعامل مؤكسد في وسط قاعدي مكوناً لوناً بني محمر • امتلكت تلك النواتج اقصى امتصاص عند الاطوال الموجية 440 و 480 و 445 نانوميتر لكل من أورثو -امينوفينول،

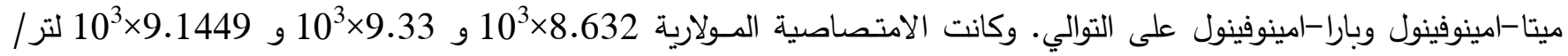
مول.سم لتراكيز اتبعت قانون بير بحدود 1-20 و 1-24 و 1-7 جزء لكل مليون للمركبات اعلاه على التوالي. اذ تراوحت دقة الطريقة بين 98.38\% و 01.01\% في حين كانت التوافقية اقل من 1.6 لجميع المركبات المدروسة. لقد وجد ان ايزومرات امينوفينول تكون

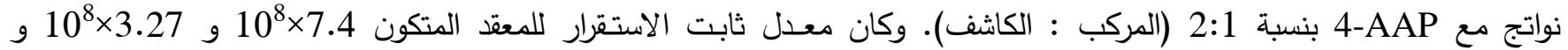

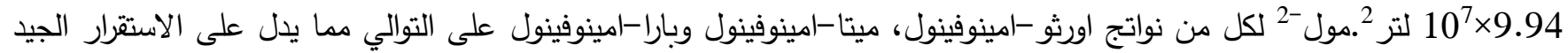

لتلك النواتج.

الكلمات المفتاحية: الأقتران التأكسدي، مطياف فوتومتري، اورثو -امينوفينول، ميتا-امينوفينول، بارا-امينوفينول، 4-امينوانتي بايرين

المقدمة

يعد الاورثو -امينوفينول(I)، والميتا-امينوفينول(II) والبارا-امينوفينول(III) من اهم المركبات الفينولية التي تحتوي على مجموعة

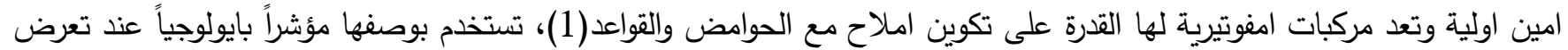
الانسان للانلين في علم السموم ولا تعد من المركبات المسرطنة(2) ولكن التعرض لها لمدة طويلة قد يسبب تلف الكبد ويؤثر على الجهاز العصبي وتصل السمية الى 40 ملغم/كغم ويعد البارا-امينوفينول ناتج ايض من الباراسيتامول(3) لذلك تدخل الامينات الفينولية في صناعة الادوية(4) واهمها الفيناستين(1) وتم اثبات سمية الامينات الفينولية على انها شبيهة بالاسيتانليد ولكن سميتها تقل عند ماتئل تدخل مجموعة حامضية على مجموعة الهيدروكسيل او مجموعة الامين، الا ان مشتقات الامينات الفينولية عامة خافضة للحمى (5)(Analgesics) ومسكنة للاعى م(Antipyretic)

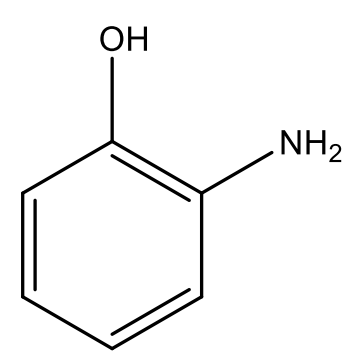

(I)

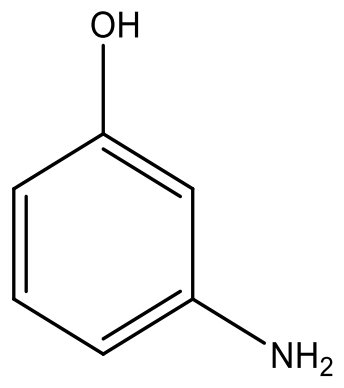

(II)<smiles>Nc1ccc(O)cc1</smiles>

(III) $\quad$ M.wt $=109.128$

تعد تفاعلات الاقتران التأكسدي من اهم التفاعلات العضوية ذات التطبيقات الواسعة خاصة في الكيمياء التحليلية، اذ يتضمن هذا التفاعل ازدواج مادتين عضويتين بوجود عامل مؤكسد تحت ظروف تفاعل مناسبة. تحدث اكسدة لهذه المواد مما يؤدي الى تكوين 


\section{Journal of Education and Science (ISSN 1812-125X), Vol: 29, No: 3, 2020 (68-84)}

مركبات وسطية تتفاعل مع بعضها وتعطي ناتج ملون يمكن قياسه طيفياً والاستفادة من التفاعل في التقدير الكمي للمركبات العضوية المختلفة(6).

تم استخدام طرق تحليلية متتوعة في تقدير ايزومرات امينوفينول منها الطرق الطيفية(7-16)، الكهربائية (17-19) والكروماتوغرافية .$(23-20)$

في هذا البحث تم تقدير ايزومرات امينوفينول طيفياً عن طريق تفاعل الاقتران التأكسدي مع كاشف 4-امينوانتي بايرين بوجود كبريتات النحاس كعامل مؤكسد وفي وسط قاعدي.

\section{الجزء العملي Experimental part}

\section{Apparatus used الأجهزة المستخدمة}

Shimadzu UV-1800 Double- تمت القياسات الطيفية بواسطة جهاز " المطياف الفوتومتري ثنائي الحزمـة من نوع

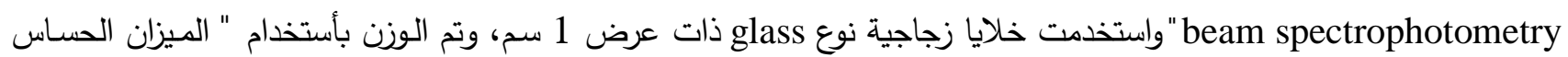
نوع (ae ADAM) وأُجريَ التسخين بأستخدام" الحمام المائي نوع (elektro.mag)"، وتم قياس حامضية المحاليل " بجهاز

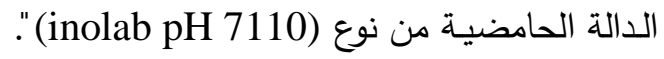
محاليل المواد الكيميائية كانت جميع المواد الكيميائية المستخدمة على درجة عالية من النقاوة. - ايزومرات امينوفينول (100 جزء لكل مليون) حضرت باذابة 0.010 غم من المادة النقية في الماء المُقطر ثم نُقلت الى قنينة حجمية سعة 100 مللتر . - 4-امينوانتي بايرين (1\%) حضرت باذابة 1.0 غرام من المادة النقية في الماء المقطر ثم نُقلت الى قنينة حجمية سعة 100 ملتر - كبريتات النحاس المائية (CuSO4.5H2O) (1) (10.1\%) حضرت باذابة 0.10 غرام من المادة النقية في الماء المقطر ثم نُقلت الى قنينة حجمية سعة 100 مللتر • - هيدروكسيد الصوديوم ( 5.0×10² مولاري) حضرت باذابة 0.50 غرام من المادة النقية في الماء المقطر ثم نُقلت الى قنينة حجمية سعة 250 مللتر . - هيدروكسيد الامونيوم (5.0×10-20 مولاري) حضرت بتخفيف 1.93 ملتتر من الامونيا المركزة (6.45 مولاري) بالماء المقطر في قنينة حجمية سعة 250 مللتر .

\section{النتائج والمناقشة : 1 - الأختبارات الأولية}

عند اضافة 1.0 مللتر من 4-امينوانتي بايرين بتركيز 1\% الى 1.0 مللتر لكل من اورثو-امينوفينول، ميتا-امينوفينول وباراامينوفينول وبتركيز 100 جزء لكل مليون وبشكل منفصل، ثم اضـافة 1.0 مللتر مـن العامل المؤكسد كبريتات النحاس المائية و 1.0 ملتر من هيدروكسيد الصوديوم لكل مركب على التوالي، وأكمل الحجم بالماء المقطر الى 25 ملتر وتركت المحاليل لمدة 10 


\section{Journal of Education and Science (ISSN 1812-125X), Vol: 29, No: 3, 2020 (68-84)}

دقائق في درجة حرارة الغرفة (30م)، وتم قياس طيف الامتصاص للمحلول البني المحمر الناتج ووجد ان شدة الامتصاص التي تصل اليها المركبات المدروسة عند الطول الموجي 440 و 480 و 445 نانوميتر للمركبات اعلاه على التوالي. 2- دراسة ظروف التفاعل المثلى

أجريت الدراسات التالية بأستخدام 4 اجزاء لكل مليون من اورثو -امينوفينول و 2 جزءان لكل مليون من ميتا-امينوفينول و 4 اجزاء لكل مليون من بارا-امينوفينول في حجم نهائي مقداره 25 مللتر. دراسة كمية كاثف الأقتران

تمت دراسة تأثير كمية الكاشف 4-امينوانتي بايرين على تكوين الناتج للمركبات المدروسة وذلك باضافة حجوم مختلفة من 4امينوانتي بايرين تتراوح بين (0.25- 4.0) مللتر تركيزه 1\% لأورثو -امينوفينول وميتا-امينوفينول وبارا-امينوفينول بشكل منفرد و و1.0 مللتر من كبريتات النحاس المائية تركيز (10.1\%) و1.0 مللتر من هيدروكسيد الصوديوم بتركيز 5 × 10-2 مولاري واكمل الحجم بالماء المقطر الى حد العلامة ثم تركت المحاليل لمدة 10 دقائق في درجة حرارة المختبر، وقيست الامتصاصات عند الأطوال الموجية 440 و 480 و 445 نانوميتر للمركبات اعلاه على التوالي، والجدول (1) يبين الحجم الافضل من الكاشف للمركبات المدروسة. الجدول (1) دراسة كمية الكاشف

\begin{tabular}{|c|c|c|c|}
\hline \multirow{2}{*}{ Ml of 4-AAP (1\%) } & \multicolumn{3}{|c|}{ Absorbance } \\
\cline { 2 - 4 } & o-amino phenol & m-amino phenol & p-amino phenol \\
\hline Without & 0.073 & 0.064 & 0.066 \\
\hline $\mathbf{0 . 2 5}$ & 0.186 & 0.098 & 0.226 \\
\hline $\mathbf{0 . 5}$ & 0.199 & 0.133 & 0.244 \\
\hline $\mathbf{0 . 7 5}$ & 0.262 & 0.265 & 0.262 \\
\hline $\mathbf{1 . 0}$ & 0.322 & 0.320 & 0.233 \\
\hline $\mathbf{1 . 5}$ & 0.342 & 0.350 & 0.209 \\
\hline $\mathbf{2 . 0}$ & 0.372 & 0.391 & 0.198 \\
\hline $\mathbf{2 . 5}$ & 0.349 & 0.307 & 0.187 \\
\hline $\mathbf{3 . 0}$ & 0.327 & 0.300 & Turbid \\
\hline $\mathbf{4 . 0}$ & Turbid & 0.252 & --- \\
\hline
\end{tabular}

تم اجراء دراسة لمعرفة تأثير العامل المؤكسد المناسب الذي يعطي اعلى امتصاص للناتج وذلك باستخدام عدد من العوامل

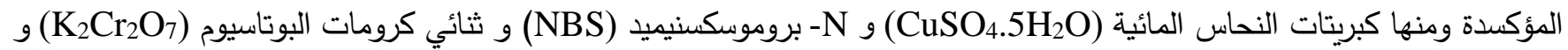

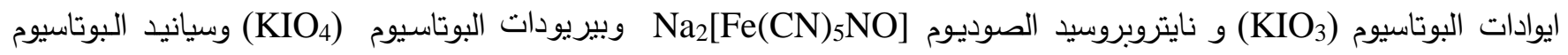
الحديد) تحتوي على المركبات المدروسة والكمية المثلى من الكاشف لكل مركب على انفراد وجعل الوسط قاعدي باضافة 1.0 مللتر من هيدروكسيد الصوديوم ثم خفقت المحاليل بـالماء المقطـر الى حد العـلامة وقيس الامتصـاص عند الاطوال الموجية المناسبة مقابل 
محساليلها الصـورية، اظهرت الدراسة ان افضل عامل مؤكسد هو كبريتات النحاس المائية (CuSO4.5H2O السابقة وتم استعماله في التجارب اللاحقة.

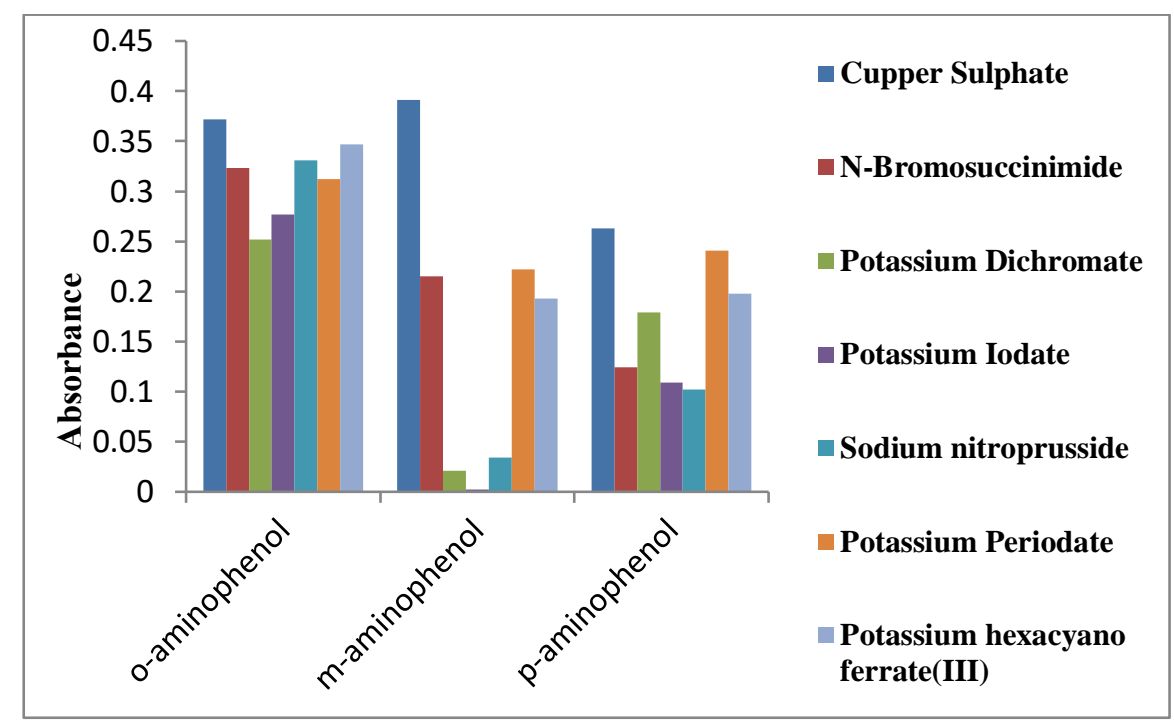

$$
\text { الشكل (1) دراسة نوع العامل المؤكسد }
$$

دراسة حجم العامل المؤكسد

تمت دراسة افضل حجم للعامل المؤكسد كبريتات النحاس المائية وذلك باستعمال حجوم متزايدة من العامل المؤكسد (0.25-2.0)

$$
\text { مللتر ، الجدول (2) يبين الحجم الامثل لكل مركب والذي تم اعتماده في التجارب اللاحقة. }
$$

\begin{tabular}{|c|c|c|c|}
\hline \multirow{2}{*}{ Ml of $0.1 \% \mathrm{CuSO}_{4.5} \mathrm{H}_{2} \mathrm{O}$} & \multicolumn{3}{|c|}{ Absorbance } \\
\hline & o-amino phenol & m-amino phenol & p-amino phenol \\
\hline Without & 0.274 & 0.023 & 0.102 \\
\hline 0.25 & 0.447 & 0.265 & 0.238 \\
\hline 0.5 & 0.450 & 0.383 & 0.326 \\
\hline 0.75 & 0.422 & 0.400 & 0.357 \\
\hline 1.0 & 0.372 & 0.391 & 0.262 \\
\hline 1.5 & 0.324 & 0.385 & Turbid \\
\hline 2.0 & 0.278 & 0.376 & --- \\
\hline
\end{tabular}

جدول (2) دراسة حجم العامل المؤكسد 
من اجل اختيار نوع القاعدة المناسبة تم دراسة عدة قواعد مختلفة مثل هيدروكسيد الصوديوم (NaOH)، هيدروكسيد البوتاسيوم

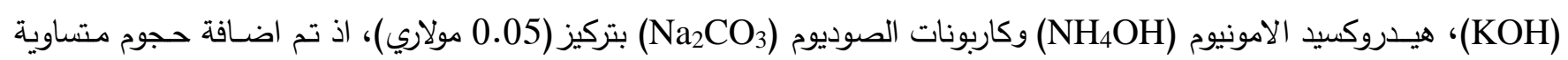

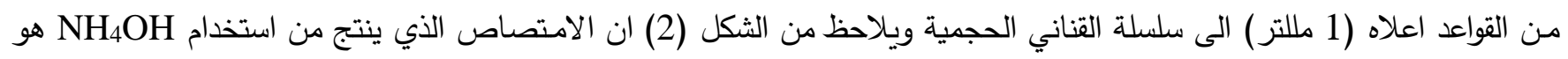
الافضل لذا تم اعتماده

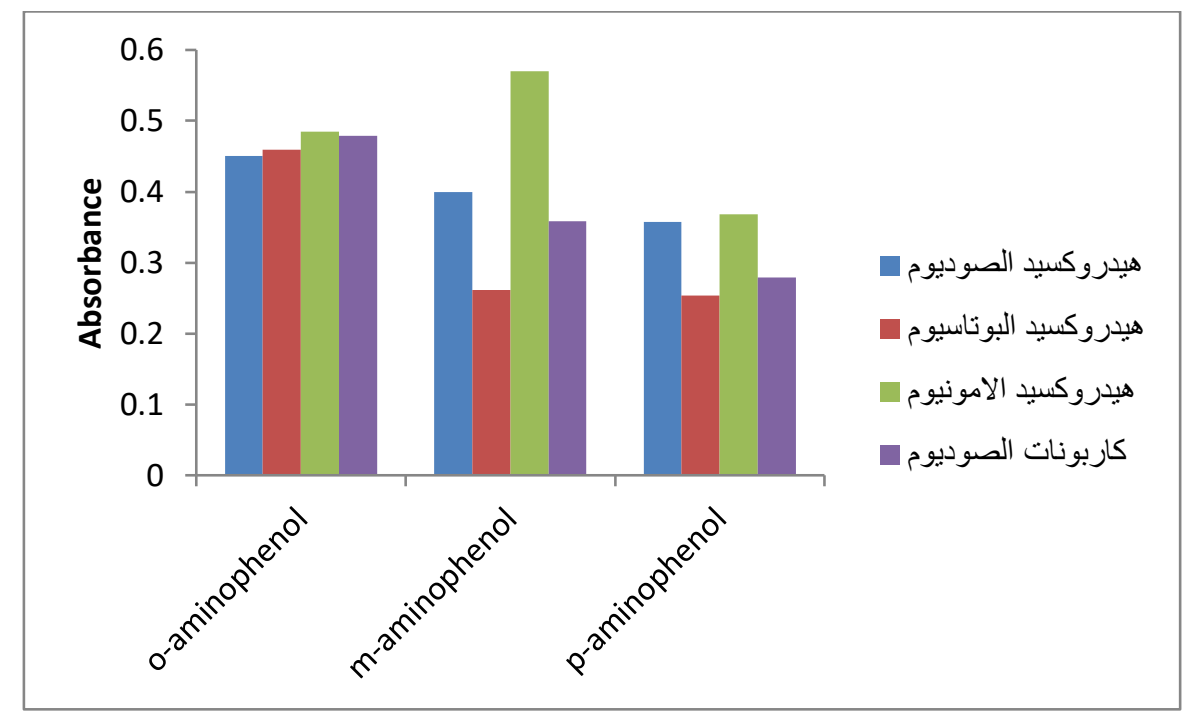

$$
\text { الشكل (2) دراسة نوع القاعدة }
$$

تأثير حجم القاعدة والدالة الحامضية

تمت دراسة افضل حجم لمحلول هيدروكيد الامونيوم بتركيز (0.05 مولاري) وقياس pH المحاليل، ويبين الجدول (3) الحجم الامثل والدالة الحامضية المثلى في التقدير وتم استخدمه في التجارب اللاحقة. جدول (3) دراسة كمية القاعدة

\begin{tabular}{|c|c|c|c|c|c|c|}
\hline \multirow{2}{*}{$\begin{array}{c}\text { Volume of 0.05M } \\
\text { NH4OH(ml) }\end{array}$} & \multicolumn{2}{|c|}{ o-amino phenol } & \multicolumn{2}{c|}{ m-amino phenol } & \multicolumn{2}{c|}{ p-amino phenol } \\
\cline { 2 - 7 } & Absorbance & pH & Absorbance & pH & Absorbance & pH \\
\hline Without & 0.368 & 7.1 & 0.491 & 7.3 & 0.286 & 7.1 \\
\hline $\mathbf{0 . 2 5}$ & 0.401 & 8.8 & 0.509 & 9.1 & 0.297 & 8.8 \\
\hline $\mathbf{0 . 5}$ & 0.409 & 9.0 & 0.533 & 9.2 & 0.352 & 9.0 \\
\hline $\mathbf{0 . 7 5}$ & 0.412 & 9.1 & 0.549 & 9.3 & 0.401 & 9.1 \\
\hline $\mathbf{1 . 0}$ & 0.485 & 9.3 & 0.570 & 9.4 & 0.368 & 9.2 \\
\hline $\mathbf{1 . 5}$ & 0.487 & 9.4 & 0.583 & 9.5 & 0.336 & 9.3 \\
\hline $\mathbf{2 . 0}$ & 0.491 & 9.6 & 0.590 & 9.6 & 0.334 & 9.6 \\
\hline $\mathbf{2 . 5}$ & 0.456 & 9.7 & 0.567 & 9.7 & 0.330 & 9.7 \\
\hline $\mathbf{3 . 0}$ & 0.400 & 10.1 & 0.535 & 9.9 & Turbid & 9.9 \\
\hline $\mathbf{3 . 5}$ & 0.393 & 11.0 & 0.500 & 10.1 & -- & -- \\
\hline
\end{tabular}




\section{Journal of Education and Science (ISSN 1812-125X), Vol: 29, No: 3, 2020 (68-84)}

تأثير الحامض والمحاليل المنطمة

تم دراسة تأثير الحامض HCl بتركيز (0.05 مولاري) وكذلك دراسة تأثير المحاليل ذات الدالة الحامضية pH = 9.1 (لمحلول

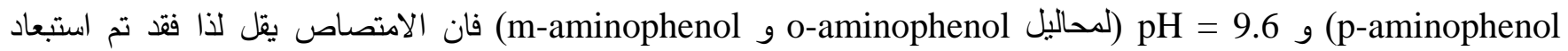
اضافة أي حامض وأي محلول منظم.

تأثير درجة الحرارة والزمن على امتصاص الناتج المتكون

درس تأثير درجة الحرارة وزمن استقرار النواتج عند درجة حرارة (30-60 مْم) بوجود كمية ثابتة من المركبات المدروسة (4 اجزاء لكل مليون لكل من o-aminophenol و p-aminophenol و2 جزءان لكل مليون من m-aminophenol) والكميات المثلى من الكاشف والعامل المؤكسد والقاعدة لكل مركب على انفراد ثم اكمال الحجم الى حد العلامة بالماء المقطر، تم قياس الامتصاص عند

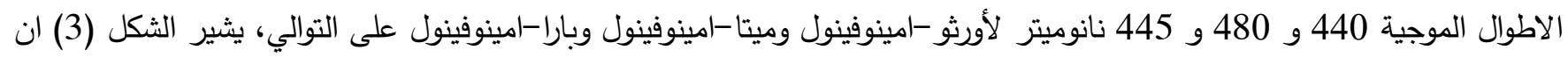

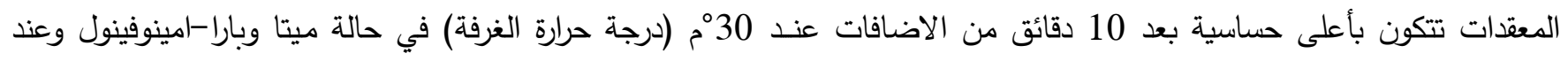
درجة حرارة 50م في حالة اورثو -امينوفينول وتبقى المعقدات مستقرة لمدة اكثر من 60 دقيقة.

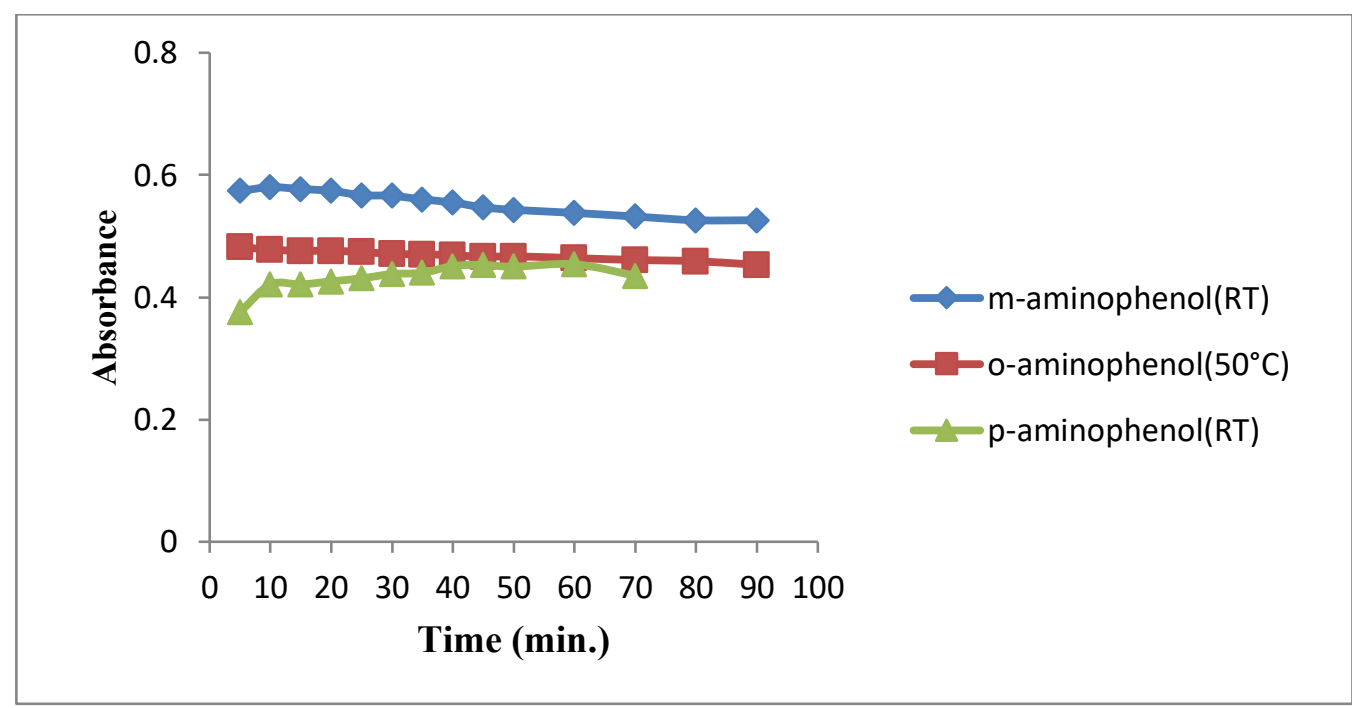

الشكل (3) دراسة درجـة الحرارة وزمن الاستقرار

دراسة تأثثير مواد الثد السطحي

لغرض زيادة الحساسية تمت دراسة عدد من مواد الثد السطحي الموجبة والسالبة والمتعادلة ووجد بان تأثيرها سلبي، اذ تعمل على تقليل قيمة الامتصـاص لذلك أستُبعدت من الدراسات اللاحقة. 


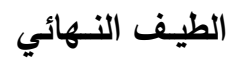

تم رسم الطيف النهائي لناتج أورثو وميتا وبارا-امينوفينول مـع الكاشف (4AP) عند اطوال موجية 350-700 نانوميتر . لقد وجد ان الت لتلك النواتج عند 440 و 480 و 445 نانوميتر للمركبات اعلاه على التوالي، الثكل (4) يوضح الرسم النهائي للمركبات المدروسة.

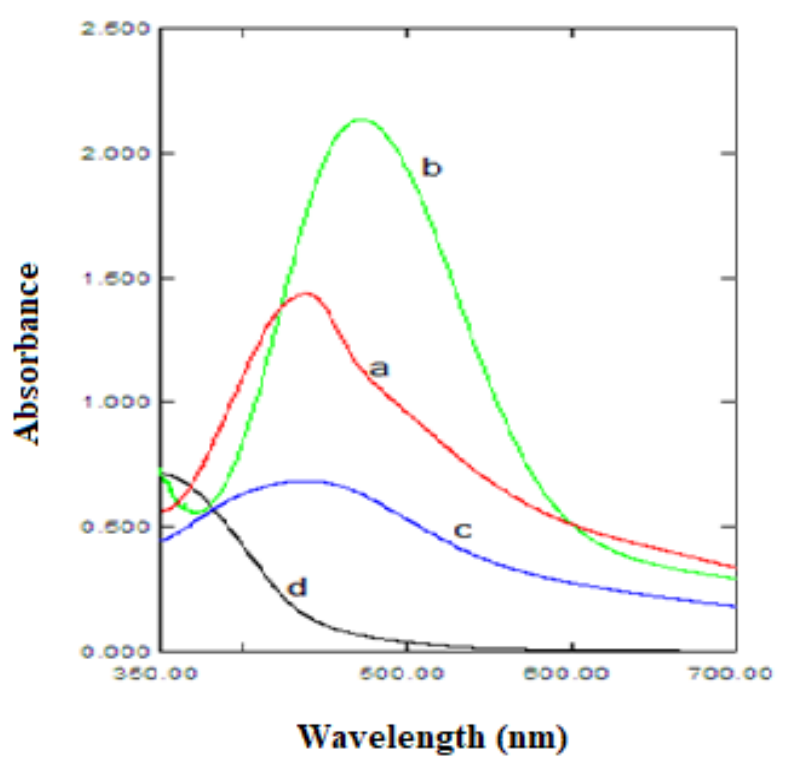

$$
\begin{aligned}
& \text { الشكل (4) الطيف النهائي }
\end{aligned}
$$

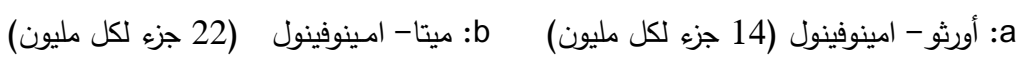

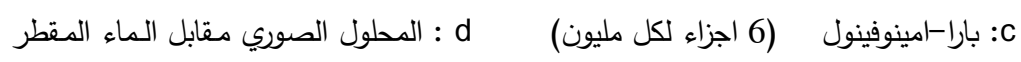

دراسة الـمنحنى الـقياسي تم الحصول على منحنيات قياسية تطيع قانون بير ضـمن التراكيز (1-20) و (1-24) و (1-7) جزء لكل مليون على التوالي، الثكل (5) لكل من اورثو-امينوفينول و ميتا-امينوفينول و بارا-امينوفينول والذي يدل على ان هناك انحرافا سلبيا بعد الحدود التقديرية

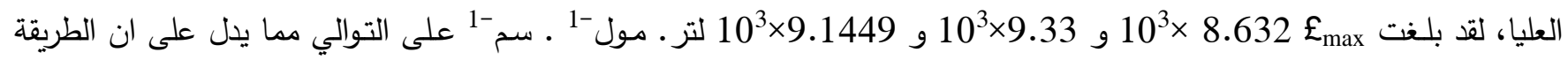
حساسة في حين بلغ حد الكثف 0.03 و 0.094 و 0.096 جزء لكل مليون والحد الكمي 0.099 و 0.3123 و 0.322 جزء لكل مليون للمركبات اعلاه على التوالي. 

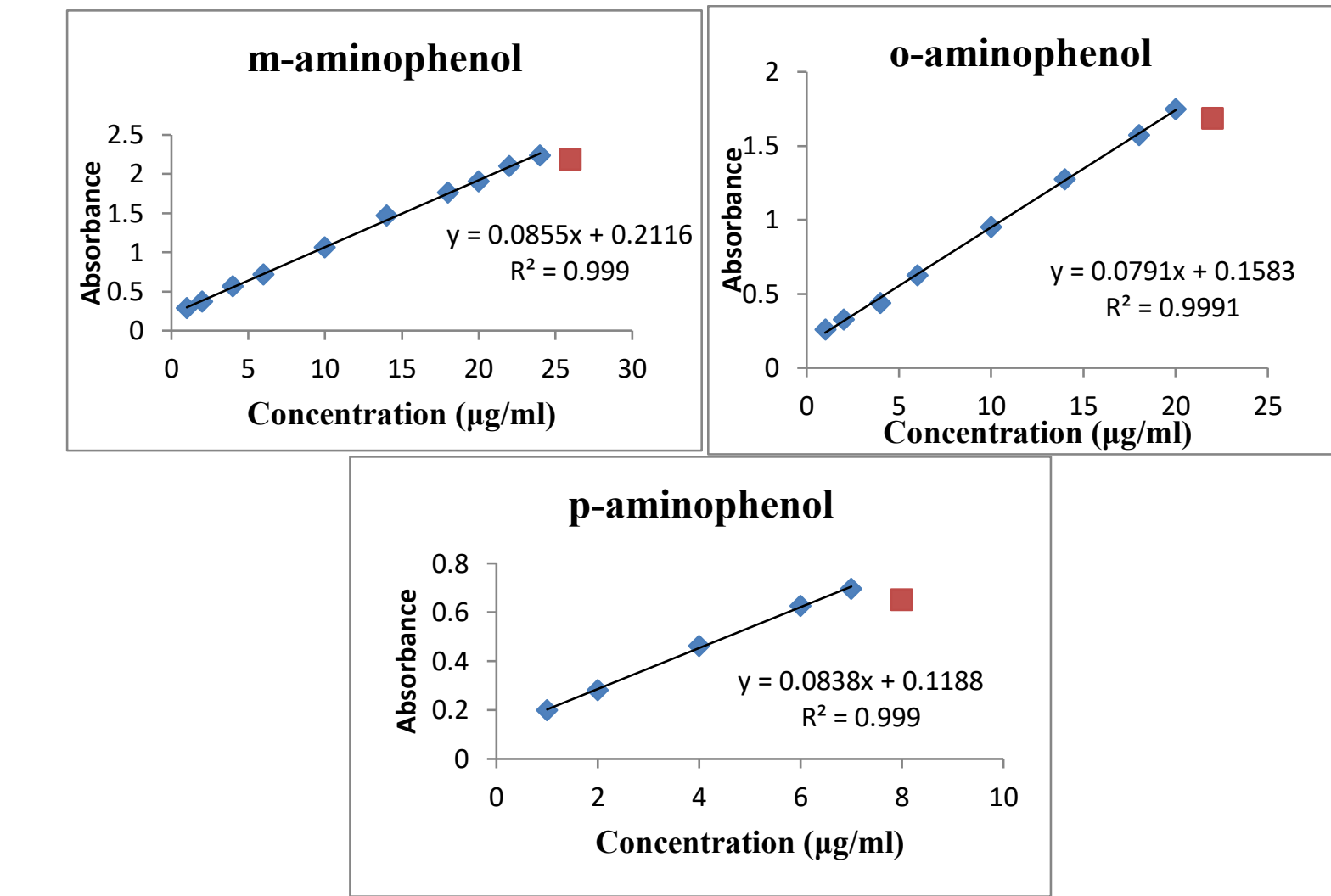

الثكل (5) المنحنيات القياسية لأيزومرات امينوفينول

الجدول (4) المواصفات الخطية والمدى التقديري والامتصاصية المولارية وحدود الكثف والتقدير الكمي في تقدير ايزومرات امينوفينول

\begin{tabular}{|c|c|c|c|c|c|c|c|}
\hline Compound & $\begin{array}{l}\text { Linearity } \\
\text { range } \\
(\text { ppm) }\end{array}$ & $\begin{array}{c}\text { "Molar } \\
\text { Absorptivity } \\
\text { (1/mol.cm)" }\end{array}$ & $\begin{array}{l}\text { "LOD* } \\
(\text { ppm)" }\end{array}$ & $\begin{array}{l}\text { "LOQ* } \\
(\text { ppm)" }\end{array}$ & Slope & Intercept & $\begin{array}{c}\text { "Correlation } \\
\text { coefficient } \\
\left(\mathbf{R}^{2}\right) "\end{array}$ \\
\hline o-aminophenol & $1-20$ & $8.632 \times 10^{3}$ & 0.03 & 0.099 & 0.0791 & 0.1583 & 0.9991 \\
\hline m-aminophenol & $1-24$ & $9.33 \times 10^{3}$ & 0.094 & 0.3123 & 0.0855 & 0.2116 & 0.999 \\
\hline p-aminophenol & $1-7$ & $9.1449 \times 10^{3}$ & 0.096 & 0.322 & 0.0838 & 0.1188 & 0.999 \\
\hline
\end{tabular}

*Average of ten determination. 
تم حساب دقـة الطريقة وتوافقها من خلال احتساب Recovery و RSD بأستخدام خمس قراءات لثلاث تـراكيز مختلفة لكل

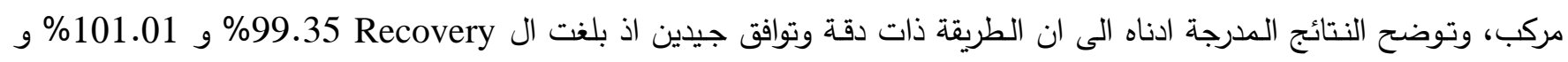

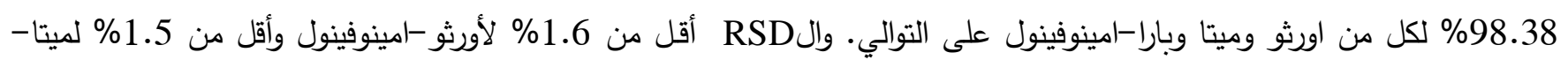

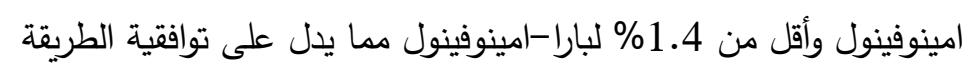

جدول (5) دقـة الطريقة وتـوافقها

\begin{tabular}{|c|c|c|c|c|}
\hline Compound & $\begin{array}{c}\text { "Amount } \\
\text { added" } \\
(\boldsymbol{\mu g} / \mathbf{m l})\end{array}$ & $\begin{array}{c}\text { "Recovery*" } \\
(\boldsymbol{\%})\end{array}$ & $\begin{array}{c}\text { "Average } \\
\text { recovery" } \\
(\boldsymbol{\%})\end{array}$ & $\begin{array}{c}\text { "RSD*" } \\
\mathbf{( \% )}\end{array}$ \\
\hline \multirow{2}{*}{ o-amino phenol } & 2 & 103.31 & & 0.71 \\
& 6 & 98.97 & 99.35 & 1.58 \\
\hline \multirow{3}{*}{ m-amino phenol } & 18 & 95.79 & & 0.42 \\
\hline & 6 & 102.82 & 101.01 & 1.16 \\
p-amino phenol & 18 & 104.64 & & 0.16 \\
& 2 & 95.59 & & 1.35 \\
& 6 & 97.65 & 98.38 & 0.70 \\
\hline
\end{tabular}

*Average of Five Determination.

تأثير طبيعة المعقد وثثابت استقراريته

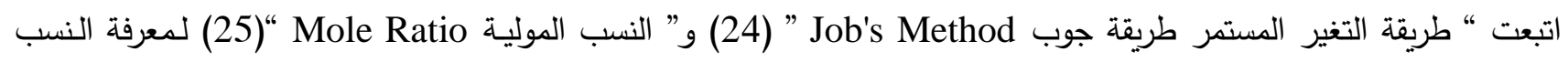

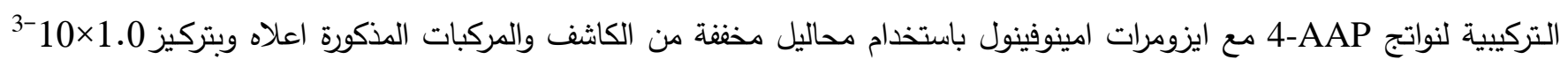
مولاري. النتائج التي تم الحصول عليها في الثكل (6) والثكل (7) تؤكد ان الناتج يتكون بنسبة 2:1 (ايزومر امينوفينول : 4-AAP) باستخدام كلتا الطريقتين. وتم احتساب ثابت استقرار النواتج المتكونة بنسبة 2:1 للمركبات اعلاه وبشكل منفصل وبتطبيق القانون التالي:

$\mathrm{K}_{\mathrm{st}}=\frac{1-\alpha}{4 \alpha^{3} c^{2}}$

حيث C هي تركيز المعقد بوحدة (مول/ لتر)، و $\alpha$ هي درجة التفكك و Kابت الاستقرار ، بلـغ معدل ثابت الاستقرار للمعقد

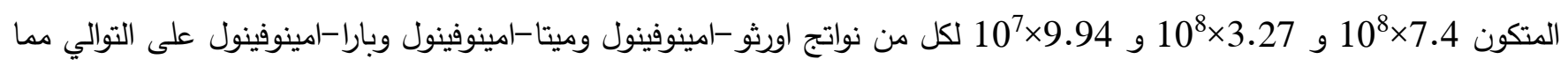

يدل على الاستقرار العالي لتلك النواتج، كما موضح في الجدول (6). 
Journal of Education and Science (ISSN 1812-125X), Vol: 29, No: 3, 2020 (68-84)

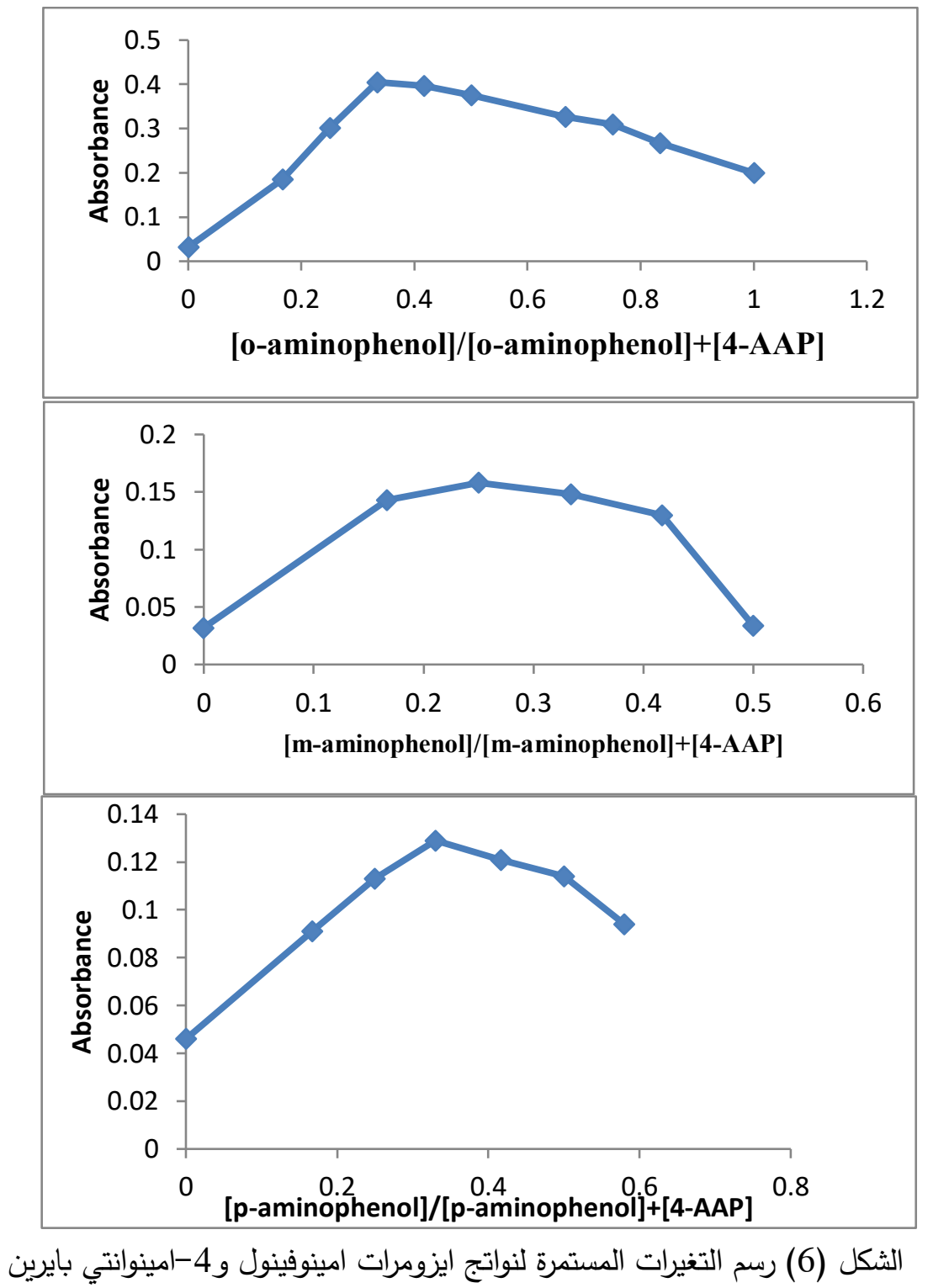


Journal of Education and Science (ISSN 1812-125X), Vol: 29, No: 3, 2020 (68-84)

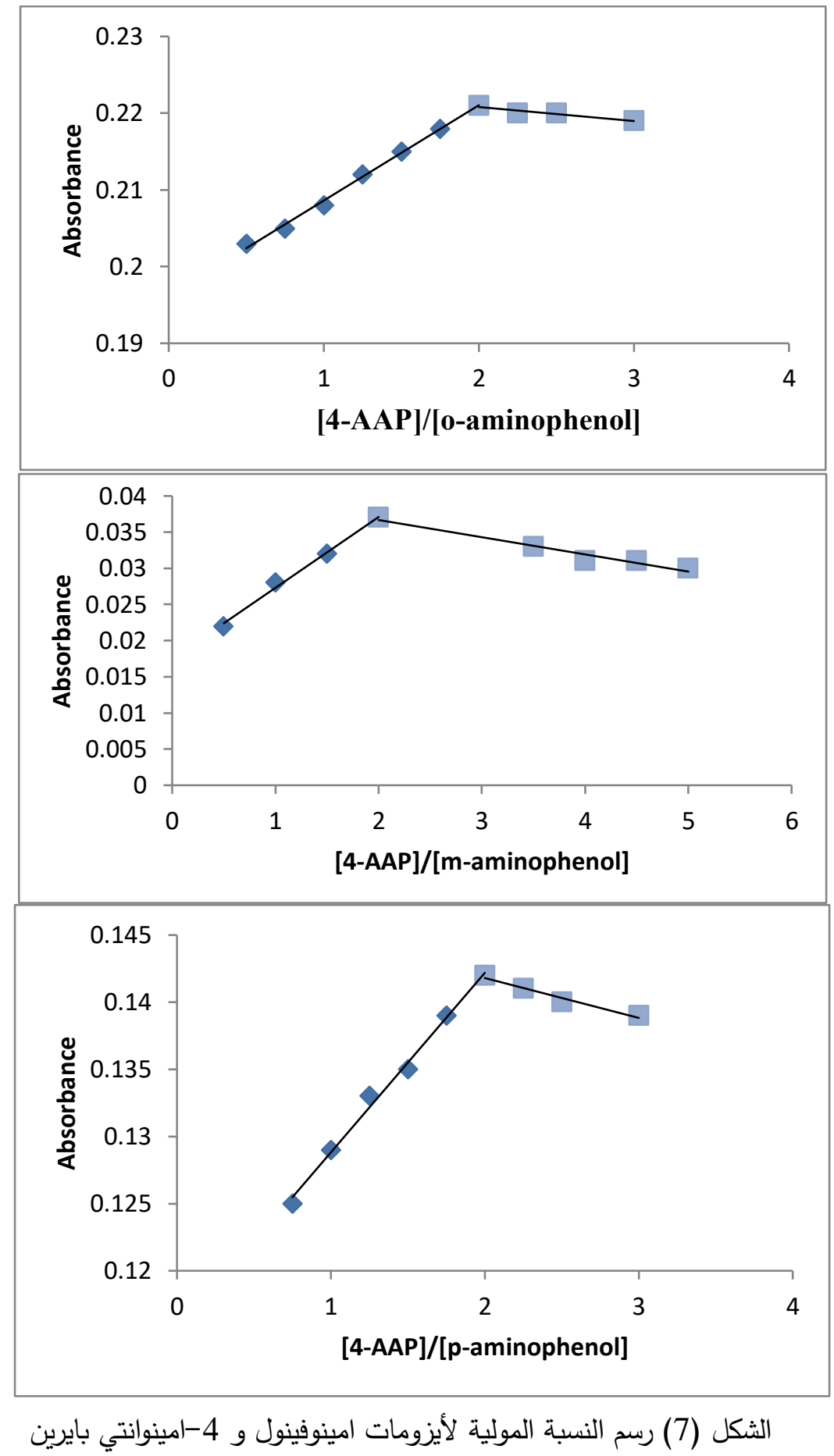


جدول (6) ثابت استقرار المعقدات مع الكاشف 4-امينوانتي بايرين

\begin{tabular}{|c|c|c|c|c|c|}
\hline \multirow{2}{*}{ Compound } & \multirow{2}{*}{$\begin{array}{l}\text { Conc. } \\
\left(\text { mol. }^{-1}\right)\end{array}$} & \multicolumn{2}{|c|}{ Absorbance } & \multirow{2}{*}{$\alpha$} & \multirow{2}{*}{$\begin{array}{c}\text { Average } K_{s t} \\
\left(l^{2} \cdot \mathrm{mol}^{-2}\right)\end{array}$} \\
\hline & & As & Am & & \\
\hline \multirow{3}{*}{ o-aminophenol } & $2 \times 10^{-5}$ & 0.161 & 0.391 & 0.588 & \multirow{3}{*}{$7.4 \times 10^{8}$} \\
\hline & $4 \times 10^{-5}$ & 0.257 & 0.506 & 0.492 & \\
\hline & $6 \times 10^{-5}$ & 0.359 & 0.712 & 0.496 & \\
\hline \multirow{3}{*}{ m-aminophenol } & $2 \times 10^{-5}$ & 0.118 & 0.363 & 0.675 & \multirow{3}{*}{$3.27 \times 10^{8}$} \\
\hline & $4 \times 10^{-5}$ & 0.245 & 0.666 & 0.632 & \\
\hline & $6 \times 10^{-5}$ & 0.343 & 0.962 & 0.643 & \\
\hline \multirow{3}{*}{ p-aminophenol } & $2 \times 10^{-5}$ & 0.044 & 0.227 & 0.806 & \multirow{3}{*}{$9.94 \times 10^{7}$} \\
\hline & $4 \times 10^{-5}$ & 0.064 & 0.374 & 0.829 & \\
\hline & $6 \times 10^{-5}$ & 0.086 & 0.520 & 0.835 & \\
\hline
\end{tabular}

دراسة الـمتداخلات

تمت دراسة عدد من المتداخلات والمتمثلة بالكحولات الاليفاتية الاولية والثانوية والمركبات الامينية الاليفاتية ومركبات عضوية

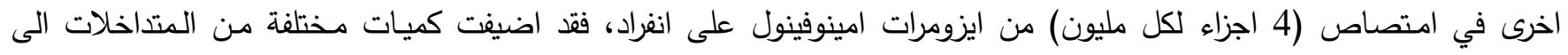

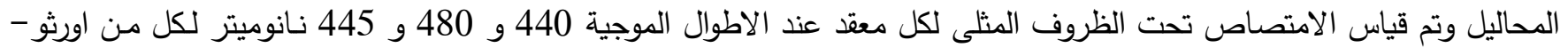
امينوفينول وميتا-امينوفينول وبارا-امينوفينول على التوالي. تثير النتائج ادناه الى انتقائية الطريقة لعدم وجود تداخل من قبل المواد جدول (7) تأثير المتداخلات السواغ المضافة.

\begin{tabular}{|c|c|c|c|c|c|c|c|c|c|c|c|c|}
\hline \multirow{3}{*}{$\begin{array}{l}\text { Foreign } \\
\text { compound }\end{array}$} & \multicolumn{12}{|c|}{ Recovery $(\%)$ of $4 \mu \mathrm{g} / \mathrm{ml}$ of aminophenol isomer per $\mu \mathrm{g} / \mathrm{ml}$ Foreign added } \\
\hline & \multicolumn{4}{|c|}{ o-aminophenol } & \multicolumn{4}{|c|}{ m-aminophenol } & \multicolumn{4}{|c|}{ p-aminophenol } \\
\hline & 100 & 500 & 750 & 1000 & 100 & 500 & 750 & 1000 & 100 & 500 & 750 & 1000 \\
\hline & 97 & 99.3 & 97.1 & & 97 & 96.3 & & & 95.6 & & & 98.4 \\
\hline Isobu & 98.2 & 98.2 & 98 & .7 & & & & 5.6 & 95.4 & & & 5.4 \\
\hline & 97.7 & 97 & 98.6 & 98.4 & 5.9 & & | & 5.3 & 97.4 & & & 7.2 \\
\hline & 95.8 & 96.6 & 97. & 95.6 & & & & 104 & 99.7 & 100 & & 98.5 \\
\hline & 101 & 97.2 & 97.4 & 98.9 & 100.9 & 102.4 & 102 & 101.5 & 97.8 & 98 . & 98.2 & 98.3 \\
\hline Sucr & 98.2 & 104 & 101 & 100.7 & 100.7 & & 99.2 & 101.2 & 96.4 & 95 . & 97.1 & 98.8 \\
\hline & 95.6 & 95.8 & 96.2 & 96.9 & 95.3 & 95.8 & 96.8 & 97.1 & 95.1 & 96.6 & 98.3 & 98.4 \\
\hline Propylamine & 99.3 & 104.3 & 104.8 & 99.5 & 95.4 & 96.1 & 97.2 & 98.8 & 103.2 & 101.9 & 101.2 & 99.8 \\
\hline
\end{tabular}


التفاعل الكيميائي المقترح

من خلال التركيب الكيميائي للمركبات العضوية (ايزومرات امينوفينول) بانها تحتوي على مجموعتي فعالة هما مجموعة الهيدروكسيل ومجموعة الأمين الاروماتية ومن خلال دراسة طبيعة الناتج المتكون بطريقتي جوب والنسبة المولية اثتت النتيجة بان الارتباط يكون بنسبة 2:1 (مركب عضوي: 4-امينوانتي بايرين) بوجود العامل المؤكسد وفي وسط قاعدي وهذا يدل على ان الارتباط يكون بمجموعة

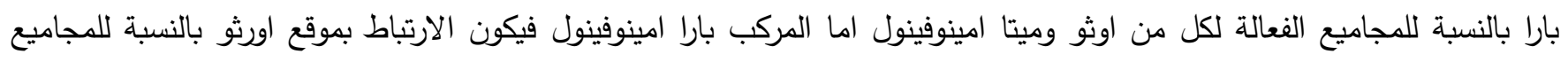
الفعالة والميكانيكية المقترحة ادناه توضح ذلك.<smiles>Nc1ccccc1O</smiles><smiles>Nc1cccc(O)c1</smiles><smiles>Nc1ccc(O)cc1</smiles>
$+$<smiles>Cc1c(N)c(=O)n(-c2ccccc2)n1C</smiles><smiles>Cc1c(N)c(=O)n(-c2ccccc2)n1C</smiles><smiles>Cc1c(N)c(=O)n(-c2ccccc2)n1C</smiles><smiles></smiles><smiles>CC(C)([14CH3])O</smiles><smiles>[13CH3]</smiles><smiles></smiles><smiles>CC1C(=O)N(c2ccccc2)N(C)C1=Nc1cc([O-])c(N=C2C(C)C(=O)N(c3ccccc3)N2C)cc1N</smiles> 


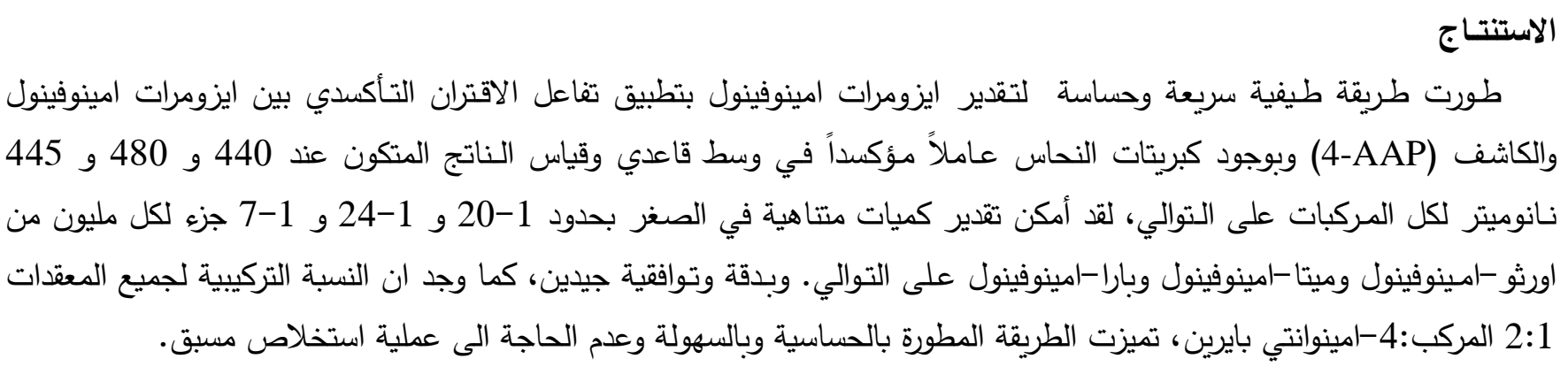

المصادر

1. J. B. Conant and A. H. Blatt, "The Chemistry of organic compounds " . $4^{\text {th }}$ Ed. Macmillan Company,New York (1982).

2. J. N. Delgado and W. A. Remers, “ Textbook of Organic Medicinal and Pharmaceutical Chemistry” . $10^{\text {th }}$ Ed. Lippincott Williams and Wilkins, Philadelphia (1998).

3. H. Song and T.S. Chen, J. Biochem. Mol. Toxicol., 15, 34-40 (2001).

4. J. H. Gaddum, "Pharmacology" $7^{\text {th }}$ Ed. Oxford University Press, London, (1975).

5. J. Crossland, “Lewis's Pharmacoloyg”. 4 ${ }^{\text {th }}$ Ed. Churchill Livingstone, Edinburgh and London (1971).

6. Grzybowski, M., Skonieczny, K., Butenschon, H. And Gryko, D. T., Comparison of Oxidative Aromatic Coupling (2013).

7. S. M. Al-Mtaiwti, "The use of charge transfer complex formation reaction in the spectrophotometric determination of some amines and drug compounds”, Ph. D. Thesis, Mosul University, p. 30, (2004).

8. H. J. Mohammed, H. J. Mohammed and H. S. Hassen, J.Islamic University., 17, 25-35, (2009).

9. N. N. Dnbeel, "Development of Spectrophotometric Methods for Determination of $o-, m$ - and $p$ Aminophenol and Their Binary Mixtures Using 1,2-Naphthoquinon-4-sulphonate-Application to Waters", M.Sc. Thesis, Mosul University, (2009). 
10. T. Al-Sabha, T. Ismeal, S. Ismael and Ali, S., Eur. Chem. Bull, 3(2),195-199 (2014).

11. E. A. Al-Hamody, "Development of spectrophotometric method for the determination of some primary amines using DNFB reagent and its applaication to metoclopramide drug", M.Sc. Thesis, Mosul University, (2005).

12. F. M. Al-Esawati, "Development of spectrophotometric methods for determination of some phenolic compounds, and drugs via organic oxdative coupling reaction”, M.Sc. Thesis, Mosul University p. 95, (2002).

13. H. Filik, M.Hayvali and E.Kilic, Anal.Chim.Acta, 535,177-182 (2005).

14. H. Filik and A. Tavman, Zh. Anal.Kh., 62,592-597 (2007).

15. N. M. L. Al-Karemy, "Development of spectrophotometric method for the determination of some amino acids and primary amines using TCNQ reagent ” M.Sc. Thesis, Mosul University, p. 60, 64 (2006).

16. N. A. F. Al-Hemiry, "Spectrophotometric electrochemical and flow injection determination of some toxics and drugs-application to various sample”,Ph.D. Thesis, Mosul University, p. 98 (2004).

17. X. Zhang, Sh. Wang and Q. Shen, Microchim.Acta, 149, 37-42 (2004).

18. Q. Chu , L. Jiang, X. Tian and J. Ye, Anal.Chim. Acta, 606,246-251 (2008).

19. W. Huang, W. Hu and J. Song, Talanta, 61, 411-416 (2003).

20. M. Narita , K. Murakami and J. Michel, Anal. Chim. Acta, 588, 316-320 (2007).

21. S. P. Wany and T. H. Huany, Anal. Chim. Acta, 534, 207-214 (2005).

22. M. S. Ali, S.Rafiuddin, M. Ghori and A. R. Kahtri, J. AOAC International (2007).

23. E. K. Wyseca, M. G. Warowna and Z. Fijalek, J.Pharm.Biomed.Anal., 32,1081-1086 (2003).

24. H. H. Willard, L. M. Merrit and J. A. Dean, "Instrumental methods of analysis", 5" ed., D. van Nostr and company NewYork, p.121 (1974). 
Journal of Education and Science (ISSN 1812-125X), Vol: 29, No: 3, 2020 (68-84)

25. L. G. Hargis, “Anaytical Chemistry” Prentice-Hall.Inc., New Jersey, p. 424-427 (1988). 